\title{
Cognitive Differentiation, Automaticity, and Interruptions of Automatized Behaviors
}

\author{
Eric J. Jolly \\ Eastern New Mexico University \\ Richard Reardon \\ University of Oklahoma
}

\begin{abstract}
The construct of field dependence-independence was explored with respect to individual efficacy in forming automatized sequences. Thirty-six female subjects developed and used such sequences. They experienced either severe, mild, or no interruption of the sequences at various points during 60 trials (such that each trial was a repetition of the sequence). Attention deployment to task-relevant and task-irrelevant material during these interruptions was assessed using recognition confidence measures. Results indicated that the distinction between task-relevant and task-irrelevant items was important to both field-dependent and field-independent subjects: Field-dependent subjects incorporated more task-irrelevant material as an attentional focus and monitored both kinds of material more closely when the sequence was not interrupted. The opposite was true for fieldindependent subjects, who were also faster in forming the sequence.
\end{abstract}

From the actions of the modern assembly-line worker to our own behavior while driving to work in the morning, "ostensibly mindless" (Langer, Blank \& Chanowitz, 1978) behavior has long been informally recognized, but only recently has it been formally examined. One approach to understanding these behaviors is through the concept of automaticity (Hasher \& Zacks, 1979; Schneider \& Shiffrin, 1977; Shiffrin \& Schneider, 1977). According to Shiffrin and

\footnotetext{
AUTHORS' NOTE: We would like to thank the editor, reviewers, and our colleague Frank Durso for their helpful comments. Preparation of the article was facilitated by a grant to the second author from the University of Oklahoma Research Associates Fund. Requests for reprints should be sent to Eric J. Jolly, Dept. of Psychology, Eastern New Mexico University, Portales, NM 88130.
}

Personality and Social Psychology Bulletin, Vol. 11 No. 3, September 1985 301-314

슬 1985 by the Society for Personality and Social Psychology, Inc. 
Dumais (1981), most processes are a mixture of automatic and controlled components. However, it is possible to classify a process or processes in terms of degree of automatism. For example, automatic processes generally occupy less cognitive capacity than controlled processes. Automatic processes may demand some attention at their initiation point but require very little attention once engaged; one must continuously attend to controlled processes (Shriffin \& Dumais, 1981).

New processes are assumed to be controlled to begin with and therefore to demand capacity. With practice, or repeated exposure, some essential target elements (those that can be represented at higher levels as units) are identified. Putting these elements into coherent units frees capacity. As Klatzky (1984) says, attention shifts from lower-order details to higher-level controlled components. To some extent, controlled processes monitor automatic ones. Periodic verification of the automatized sequence is necessary for the information-processing system to decide to continue the sequence. Interruption of the automatized sequence brings a return of controlled processing to the lower-level details (Klatzky, 1984; Schank \& Abelson, 1977; Shiffrin \& Dumais, 1981; Terry, Samuels, \& LaBerge, 1976). We can, for example, maintain a conversation with a friend while following our often-traveled route from office to library. We no longer have to attend to all of the details of the route as we did the first time we traveled it. If new construction or another obstacle were to appear along the route, we would again have to attend to the details of traveling and our conversation would suffer.

Thus automaticity is a function of our need to direct attention and effort in optimal ways. We can complete some mindless task and at the same time deploy attention to some other more effortful undertaking. Relatively little attention is directed at the elements of the automatized task and more attention is directed toward external elements. 
Little attention has been paid to the issue of individual efficacy in the formation and use of automatized sequences, through certain aspects of the automaticity concept would seem to bear scrutiny from an individual differences perspective. For instance, following the discussion and research cited above, it appears that automaticity requires an ability to isolate essential elements of the behavior to be automatized so that they can be utilized; a certain flexibility in attention deployment so that as the elements of the automatized sequence are verified, attention can be paid to external elements; and a reorientation of attention to the automatized sequence (i.e., a return to controlled processing of the lower-level elements) following failures to verify. However, individuals can vary in their ability to isolate essential stimulus elements and in the flexibility of their attention deployment. Because it attempts to account for these kinds of individual differences, the field dependenceindependence dimension (Witkin, Goodenough, \& Oltman, 1979) may provide a particularly suitable starting point for looking at the role of personality in the formation and use of automatized sequences.

Field dependence-independence is a continuum within the larger construct of psychological differentiation. Less differentiated individuals are said to approach the world in a global, field-dependent (FD) manner; more differentiated individuals approach the world in an analytical, fieldindependent (FI) manner. According to Witkin et al. (1979), the tendency to function in a field-dependent or independent fashion is manifest in all of an individual's psychological and neurophysiological activities.

FD persons tend to accept the organization of a field as given. In concept attainment situations, they sample less fully from the available cues in the environment, being dominated by the more salient ones. When the salient cues happen to be more relevant cues for solution of the concept attainment problem, this tendency on the part of FD persons 
is highly adaptive. When less salient cues are more relevant to the solution, FI persons have the adaptive advantage (Goodenough, 1976). FI persons have demonstrated a superiority at isolating and encoding the essential elements of memory tasks (Davis \& Frank, 1979; Bennink \& Spoelstra, 1979; Reardon \& Rosen, 1984). Some of these memory differences have been related to the demonstrated tendency of FD subjects to be more "in touch" with the sensory, semantic, and contextual details of the memory stimuli than FI subjects, the latter being more aware of their own cognitive processes (Durso, Reardon, \& Jolly, 1985). Consistent with this point, others have founded that "fielddependent individuals have stronger associations linking 'nonessential' or contextual features of category members to their categories than do field-independent individuals" (Nahinsky, Morgan, \& Oeschger, 1979, p. 502). Finally, FD persons are more easily distracted during learning and memory tasks (Konstadt \& Forman, 1965) and are less flexible in attention deployment than FI persons (Mendelsohn, Griswold, \& Anderson, 1966).

We can summarize the above discussion of cognitive differentiation in terms of its impact on the formation of automatized sequences by outlining our predictions. Our first task was simply to demonstrate that the automaticity and differentiation concepts can be tied together. The ability of FI persons to isolate the essential relevant items of a task should allow them to automatize the sequence more efficiently, keying on fewer elements for verification. That is, once an automatized sequence is engaged, they do not have to attend to as many elements to verify and maintain the sequence. Our second task was to demonstrate that cognitive differentiation is related to selective attention to available stimuli. Because they are less flexible in attention deployment, FD persons were expected to attend relatively more to elements of the automatized sequence, being less 
selective about whether that material is relevant to completion of the sequence. Given interruption of the automatized sequence, FD persons were expected to divert attention to the distraction, which has become the most salient feature in the environment, as evidenced by lesser attention to elements of the automatized sequence. FI persons, who have been attending less to the sequence elements, were expected to reorient to those elements, and to do so selectively by focusing on the relevant versus irrelevant material. Finally, as the level of interruption became more severe, the effects above attributed to interruption were expected to be magnified.

Our subjects were presented with a novel set of subtasks in the context of a situation that led them to automatize these subtasks. In addition to allowing us to look directly at the development of the automatized sequence, our procedure let us control the timing and severity of the interruption and provided us with readily identifiable task-relevant and taskirrelevant material.

\section{METHOD}

Subjects

The subjects were 37 female undergraduates who participated as one option of a research familiarization requirement for introductory psychology courses.

\section{Procedure}

All subjects were recruited under the guise of participating in a norming experiment designed to assess the cross-test reliability of a series of psychological instruments. The purpose of this misdirection was to ensure that the later memory measures would be truly incidental. 
Subjects were run individually. Upon arrival at the lab, each subject was seated at a table facing the blank wall of a partitioned laboratory. She was given the Group Embedded Figures Test ([GEFT] Witkin, Oltman, Raskin, \& Karp, 1971) to assess degree of field dependence-independence. Following completion of the GEFT, the subject was given a filler task (a word checklist and the Shipley-Hartford Verbal Intelligence Scale; See Sines, 1958). These tasks lent credibility to the deception, provided a potential verbal ability covariate, and allowed the experimenter time to score the GEFT and record the results.

Once the GEFT and distractor tasks were completed, the subject was told that there would be a brief delay following which she would be given another series of tests that were similar to the ones she had just finished. The purpose of the delay, she was told, was to assess the test-to-test reliability of the instruments. Then the experimenter excused himself, explaining that he would be using the delay to prepare for a different, unrelated experiment. The experimenter moved to the section of the laboratory behind the partition to begin this task. The subject was left in the generally nondescript section of the lab, waiting for the retest portion of her experiment.

While busily "preparing materials" for this "other" experiment, the experimenter looked from around the partition and offered the subject the opportunity to pass the time by helping to set up some response sheets for this "other" experiment. ${ }^{1}$ After accepting the invitation, the subject joined the experimenter in the second section of the laboratory where she was seated before a video monitor.

The subject was given a brief description of an experiment in "cross-modal processing" and informed that she would be helping put together the visual component of that experiment. Meanwhile, she was told that the experimenter would be putting together the accompanying verbal section. The 
seating arrangement for experimenter and subject was such that the latter was never under conspicuous observation of the former.

The tasks assigned to the subject involved the following elements: (1) to remove a scoring sheet from a prepared packet; (2) to place this sheet on a tray in front of the video monitor; (3) to record on the response sheet three letters (CVC trigrams), which were located within three of seven numbered columns at the base of the televised display (taskrelevant material); (4) to record the location of a key figure (an arrow) within an array of nine geometric forms on the display (task-irrelevant material); and (5) to place the response sheet in a file area to her left. The display was then changed, a new trigram appeared in different columns, and a new arrangement of geometric figures appeared (two of which were novel) with a relocated arrow. The above series of tasks was then repeated at 10 -second intervals ${ }^{2}$ for a total of 60 trials. This highly repetitious behavior was assumed to be automatized when the subject could perform all elements of a trial before a display change.

As the subject was performing the automatized sequence, the experimenter pretended to attend to a series of aural stimuli. The aural material involved a collection of highly stereotypic sentences in which the last word was provided by a different person than was heard reading the rest of the sentence. For example, "Frozen water is called ice." (See Durso et al., 1985, for details regarding these stimuli.) These sentences were synchronized with the visual display, one sentence for each trial. While ostensibly recording responses for these sentences, the experimenter was able to covertly record the trials-to-criterion for the development of the subject's automatized sequence. Thus from the subject's view, she was working with the experimenter, killing time by preparing a few answer sheets in a place marked "for experimenter's use only," as the experimenter prepared related 
verbal material. Each subject had been informed that she could feel free to discard any misprinted or mismarked coding sheets.

At three points during the course of the 60 trials, the subject experienced severe, mild, or no interruption of the sequence. At the point designated for no interruption, the script proceeded in the usual, routine fashion. The nointerruption trials were thus representative of the average automatized trial. In the mild interruption condition, it was arranged for the subject to discover a blank response sheet, in which case she was to put it aside and go on to the next sheet in her packet. In the severe interruption condition, not only did the subject discover a blank response sheet, but she was faced with a brief flashing of the video screen. That is, the severe interruption condition included the same problem as mild interruption, with the added inconvenience of a flashing screen. The type and location of these interruption or no-interruption situations were counterbalanced across subjects. Each subject experienced all three conditions. The locations of the possible interruptions were at the 19th, 32nd, and 49th trials.

At the end of the procedure, each subject was asked to indicate her confidence that a particular trigram or geometric form appeared on the monitor. Recognition confidence was chosen as the appropriate measure after pilot work showed that subjects found simple "hit-miss" recognition very difficult, resulting in rather unstable, uninformative data (i.e., a "floor effect"). The target items were those found in the trials immediately following the interruption manipulation trials. The trigrams had been matched for equally higher Archer values at these points (Archer, 1960). The target geometric forms were from the two novel forms presented in the key trials. The subject was also asked to indicate her confidence that a particular word had been heard to assess the extent to which the experimenter's task may have con- 
founded the results. All confidence ratings were made on a 6-point scale, ranging from 1 = "positively no" to $6=$ "positively yes." The stimulus materials were presented among a list of foils, that is, trigrams, forms, and words that did not appear in any trial.

Before being dismissed, all subjects were questioned as to the effectiveness of the cover story. Apparently none of them saw through the misdirection.

\section{RESULTS}

\section{Development of the Automatized Sequence}

Subjects were assigned to FD and FI groups via a tertile split on GEFT scores (no FD subject had a score higher than 9; no FI subject had a score lower than 11; and the orders of presentation of the interruption manipulation were represented equally often in both groups after the split). Thus there were 12 subjects in each group.

Once a subject could perform an entire sequence within an allotted 10-second trial, she was said to have automatized the task. The mean number of trials-to-criterion for FD subjects was 12.8; for FI subjects it was 3.8. This greater efficiency on the part of FI subjects was significant $[\mathrm{t}(23)=4.16, \mathrm{p}<.001$, two-tailed].

Preliminary analyses of covariance, with ShipleyHartford verbal intelligence as the covariate, were performed on all measures. Verbal ability did not appreciably affect any pattern of results. Thus only the $2 \times 3$, FD-FI $\times$ level of interruption, mixed-model analyses of variance, with level of interruption as the repeated-measures factor, are reported below. To ensure that there were no FD-FI false positive response biases affecting our results, the false positive confidences of FD and FI groups were compared for both task-relevant and task-irrelevant material. There were 
TABLE 1 Mean CVC (Task-Relevant) Recognition Confidence

\begin{tabular}{lcccr}
\hline & \multicolumn{4}{c}{ Level of Interruption } \\
\cline { 2 - 5 } & None & Mild & Severe & $M$ \\
\hline Field-dependent & 4.25 & 2.25 & 2.92 & 3.14 \\
Field-independent & 2.17 & 3.13 & 3.75 & 3.02 \\
M & 3.21 & 2.69 & 3.34 & \\
\hline
\end{tabular}

no significant differences in either case $[\mathrm{t}(23)=1.54$ and 0.42 , respectively, ps $>.10$, two-tailed].

In the analyses of the aural word recognition confidence, no effects approached significance. Apparently subjects were able to successfully keep this task separate from their own task.

\section{Task-Relevant Material}

Mean CVC trigram confidence scores are given in Table 1. There was one significant effect, a FD-FI $\times$ level of interruption interaction $[\mathrm{F}(2,44)=5.10, \mathrm{p}<.02]$. FD subjects showed better recognition confidence than FI subjects with no interruption. With a mild interruption, the recognition confidence of FD subjects dropped dramatically as that of FI subjects improved. These trends were maintained in the severe initiation conditions.

\section{Task-Irrelevant Material}

Recognition confidence scores for the two geometric forms were virtually identical, so a combined geometric form confidence score was computed by summing the two separate scores. The means for these sums are given in Table 2. The analysis of variance on the sums revealed a main effect for level of interruption. As the interruption became more severe, recognition confidence declined $[\mathrm{F}(2,44)=4.71, \mathrm{p}<$ .02]. Although the interaction was not significant, Table 2 suggests that most of the decline can be accounted for by the 
TABLE 2 Mean Geometric Form (Task-Irrelevant)

Recognition Confidence (sum of two figures)

\begin{tabular}{lcccr}
\hline & \multicolumn{4}{c}{ Level of Interruption } \\
\cline { 2 - 5 } & None & Mild & Severe & $M$ \\
\hline Field-dependent & 8.42 & 7.25 & 5.42 & 7.03 \\
Field-independent & 6.08 & 6.00 & 5.42 & 5.83 \\
M & 7.25 & 6.63 & 5.42 & \\
\hline
\end{tabular}

FD group, the decline by the FI group being slight by comparison. Post hoc simple-effects analyses showed that the effect of level of interruption was significant when considering only $F D$ subjects $[F(2,44)=6.21, p<.01]$, but not when considering only $\mathrm{FI}$ subjects $(\mathrm{F}<1.0)$. Thus the pattern of recognition confidence found for the task-relevant measure was not repeated with the task-irrelevant measure: FI subjects did not show better recognition confidence for taskirrelevant material with increasing severity of interruption.

\section{DISCUSSION}

The significant relationship between FD-FI and trials to mastery of the automatized sequence is evidence that cognitive differentiation may be fundamentally related to automaticity. FI subjects were more efficient in forming the sequence. This speed of mastery may be indicative of differences in style of organizing and relating to the elements that comprise the automatized-task. An inspection of our measures of memory confidence for task-relevant and taskirrelevant material gives us some clues about these possible differences.

Looking first at the task-relevant material (CVC trigrams), FD subjects were monitoring this material closely during the uninterrupted sequences, whereas FI subjects were not. However, when the sequence was interrupted the 
patterns for recognition confidence changed. During interrupted sequences, FI subjects monitored the material more closely than FD subjects, as measured by their superior recognition confidence. This superiority was found for both levels of interruption. These results support our belief that FD persons need to refer often to task-relevant material to maintain an uninterrupted sequence, whereas FI persons need to refer to it less often. With interruption, FD persons are unable to monitor the material as closely, or choose not to do so. FI persons reorient to the material.

Consider now the task-irrelevant material (geometric forms). Both groups showed decreasing attention to the material as the level of interruption increased. However, post hoc analyses indicated that most of this decline in attention was due to the FD subjects. Importantly, FI subjects did not show the same reorientation to task-irrelevant material with interruption that they showed with task-relevant material. This supports our contention that FI subjects are better able to distinguish between task-relevant and irrelevant material. When the sequence is interrupted, the FI subjects seem to reorient only to the relevant, essential items. FD subjects do not make this distinction; the breakdown in attention for them affects relevant and irrelevant material in a similar manner. FD subjects appear to have a broader, less efficient focus on task material; FI persons have a narrow, efficient focus.

Using automaticity as a perspective for viewing cognitive differentiation has helped us gain a better understanding of some of the attentional consequences of being fielddependent or field-independent. In turn, using cognitive differentiation as a perspective for viewing automaticity, we may have gained a better understanding of the latter. Had we not looked at the automaticity-differentiation link, we could only have concluded from our data that automatized sequence interruptions have a deleterious effect principally on 
material that is less relevant to the sequence. The personsituation interaction for relevant material shows that this is clearly not the case. Finally, the study gives new meaning to the term "distraction" with respect to automatized sequences. For FD persons, a distraction is really a distraction; for FI persons, a distraction may actually increase attention to the critical elements of the sequence.

\section{NOTES}

1. Only one subject refused to help the experimenter. This subject's GEFT score (10) was close to the median. She was given credit and dismissed from further participation, resulting in a final sample size of 36.

2. Based on pilot work, 10 seconds was found to be the optimal time for subjects to complete the task, after a number of trials and without leftover "dead" time.

\section{REFERENCES}

Archer, E.J. (1960). A re-evaluation of meaningfulness of all possible CVC trigrams. Psychological Monographs, 74, 1-23.

Bennink, C. D., \& Spoelstra, T. (1979). Individual differences in field articulation as a factor in language comprehension. Journal of Research in Personality, 13, 480-489.

Davis, J. K., \& Frank, B. M. (1979). Learning and memory of field independentdependent individuals. Journal of Research in Personality, 13, 469-479.

Durso, F. T., Reardon, R., \& Jolly, E.J. (1985). Self-nonself segregation and reality monitoring. Journal of Personality and Social Psychology, 48, 447-455.

Goodenough, D. R. (1976). The role of individual differences in field dependence as a factor in learning and memory. Psychological Bulletin, 83, 675-694.

Hasher, L., \& Zacks, R. T. (1979). Automatic and effortful processes in memory. Journal of Experimental Psychology: General, 108, 365-388.

Klatzky, R. L. (1984). Memory and awareness: An information processing perspective. New York: Freeman.

Konstadt, N., \& Forman, E. (1965). Field dependence and external directedness. Journal of Personality and Social Psychology, 1, 490-493.

Langer, E. J., Blank, A., \& Chanowitz, B. (1978). The mindlessness of ostensibly thoughtful action. Journal of Personality and Social Psychology, 36, 635-642.

Mendelsohn, G. A., Griswold, B. B., \& Anderson, M. L. (1966). Individual differences in anagram-solving ability. Psychological Reports, 19, 799-809. 
Nahinsky, I. D., Morgan, M.S., \& Oeschger, D.E. (1979). Cognitive strategies, field dependence, and the abstraction process. Journal of Research in Personality, 13, 490-504.

Reardon, R., \& Rosen, S. (1984). Psychological differentiation and the evaluation of juridic information: Cognitive and affective consequences. Journal of Research in Personality, 18, 195-211.

Schank, R. C., \& Abelson, R. P. (1977). Scripts, plans, goals, and understanding. Hillsdale, NJ: Lawrence Erlbaum.

Schneider, W., \& Shiffrin, R. M. (1977). Controlled and automatic human information processing I: Detection, search and attention. Psychological Review, $85,1-66$.

Shiffrin, R. M., \& Dumais, S. T. (1981). The development of automatism. In J. R. Anderson (Ed.), Cognitive skills and their acquisition. Hillsdale, NJ: Lawrence Erlbaum.

Shiffrin, R. M., \& Schneider, W. (1977). Controlled and automatic human information processing II: Perceptual learning, automatic attending, and a general theory. Psychological Review, 84, 127-190.

Sines, L. K. (1958). Intelligence test correlates of Shipley-Hartford performance. Journal of Clinical Psychology, 14, 399-404.

Terry, P., Samuels, S. J., \& LaBerge, D. (1976). The effects of letter degradation and letter spacing on word recognition. Journal of Verbal Learning and Verbal Behavior, 15, 577-585.

Witkin, H. A., Goodenough, D. R., \& Oltman, P. K. (1979). Psychological differentiation: Current status. Journal of Personality and Social Psychology, 37, 1127-1145.

Witkin, H. A., Oltman, P. K., Raskin, \& Karp (1971). A manual for the embedded figures test. Palo Alto, CA: Consulting Psychologists Press.

Eric J. Jolly is Assistant Professor of Psychology at Eastern New Mexico University. His research interests are in the areas of social cognition and cognitive style.

Richard Reardon is Assistant Professor of Psychology at the University of Oklahoma. His research interests are in social cognition/social perception and cognitive psychology. 